\title{
ANÁLISE DE AGRUPAMENTO DA VEGETAÇÃO DE UM FRAGMENTO DE FLORESTA ESTACIONAL DECIDUAL ALUVIAL, CACHOEIRA DO SUL, RS, BRASIL
}

\section{CLUSTER ANALYSIS OF THE VEGETATION OF A FRAGMENT OF SEASONAL DECIDUOUS ALLUVIAL FOREST IN CACHOEIRA DO SUL, RS, BRAZIL}

\author{
Maristela Machado Araujo ${ }^{1}$ Solon Jonas Longhi ${ }^{2}$ Doádi Antônio Brena ${ }^{3}$ \\ Paulo Luiz Contente de Barros ${ }^{4}$ Sílvio Franco ${ }^{5}$
}

\begin{abstract}
RESUMO
No estado do Rio Grande do Sul, as florestas ripárias se encontram alteradas pela ação antrópica formando fragmentos. O estudo teve como objetivo analisar a estrutura e florística interna dessas florestas o que subsidiará informações para o restabelecimento desses ecossistemas. A área escolhida foi um fragmento (3004'36”'S; 52 53'09”W), de 4 ha, localizada no município de Cachoeira do Sul, RS, no Baixo Rio Jacuí. As espécies arbóreas, arbustivas e lianas (somente quanto à forma de vida) foram inventariadas, utilizando-se faixas perpendiculares ao rio, distanciadas por $50 \mathrm{~m}$, as quais apresentaram $10 \mathrm{~m}$ de largura e comprimento que variou com a largura da floresta. As faixas foram divididas em unidades amostrais de $10 \mathrm{x}$ $10 \mathrm{~m}$, nos quais foram identificados indivíduos com circunferência a $1,3 \mathrm{~m}(\mathrm{CAP}) \geq 15 \mathrm{~cm}$, registrados os valores de circunferência e altura. Os dados de densidade por espécie formaram uma matriz (70x42) utilizada na análise multivariada. A presença de agrupamentos de espécies no interior do fragmento foi avaliada pelo TWINSPAN (Two-way indicator species analysis), com base no qual foi constatada a existência de três subformações florestais $\left(S-F_{1}, S-F_{2}\right.$ e $\left.S-F_{3}\right)$. A $S-F_{1}$ foi caracterizada por ter maior influência das enchentes e lençol freático mais próximo da superfície; a $\mathrm{S}-\mathrm{F}_{2}$ ocorreu na parte central do fragmento, mas apresentou forte influência dos extravasamentos causados pelas enchentes; e na $\mathrm{S}-\mathrm{F}_{3}$, também na porção central, ocorreu maior influência do lençol freático. As espécies indicadoras das subformações foram: Sebastiania commersoniana e Eugenia uniflora ( $\left.\mathrm{S}-\mathrm{F}_{1}\right)$; Gymnanthes concolor, Cupania vernalis e Seguieria aculeata $\left(\mathrm{S}-\mathrm{F}_{2}\right)$; e Casearia sylvestris e Allophylus edulis $\left(\mathrm{S}-\mathrm{F}_{3}\right)$. Portanto, em projetos de preservação, conservação e restabelecimento desses ecossistemas, a comunidade florestal não pode ser tratada unicamente como ripária, mas considerando as variações ambientais e, conseqüentemente, florísticas.
\end{abstract}

Palavras-chave: floresta ripária; fragmento; análise de agrupamentos; subformações.

\footnotetext{
ABSTRACT

In the state of Rio Grande do Sul, the riparian forests have been altered and fragmented by human activity. The objective of this study was to analyze the structure and floristic composition of these forests, therefore providing information for the re-establishment of these ecosystems. The study site is a 4 ha fragment located in Cachoeira do Sul- RS, low Jacuí River (30'04'36”'S; 52 53'09'W). The arboreal and shrubby species and lianas (only life-form) were inventoried using $10 \mathrm{~m}$ wide strips that were laid out perpendicular to the river and spaced at $50 \mathrm{~m}$ intervals. The strips were divided into $10 \mathrm{x} 10 \mathrm{~m}$ plots. Within each plot all a individuals with girth at breast height $-\mathrm{SBH} \geq 15$ were identified and their circumference and height measured. Species density data were entered into a matrix ( $70 \times 42)$ that was used for multivariate analysis. The presence of species clusters at the interior of the fragment was analysed with TWINSPAN (Two-way indicator species analysis), which identified the existence of three forest sub-

1. Engenheira Florestal, Dra ${ }^{\mathrm{a}}$, Pesquisadora do Projeto MANFLORA, Universidade Federal Rural da Amazônia/Universidade da Flórida, Tv. Maurití, 3269, Bloco B, Apto. 904, Bairro Marco, CEP 66077-530, Belém (PA). maristela@ufra.edu.br

2. Engenheiro Florestal, Dr., Professor Titular do Departamento de Ciências Florestais, Centro de Ciências Rurais, Universidade Federal de Santa Maria, CEP 97105-900, Santa Maria (RS). longhiso@ccr.ufsm.br

3. Engenheiro Florestal, Dr., Professor aposentado do Departamento de Ciências Florestais, Centro de Ciências Rurais, Universidade Federal de Santa Maria, CEP 97105-900, Santa Maria (RS). brena@ ccr.ufsm.br

4. Professor do Departamento de Ciências Florestais, Universidade Federal Rural da Amazônia, CEP 66077-530, Belém (PA).

5. Engenheiro Agrônomo, Cachoeira do Sul (RS)

Recebido para publicação em 2/6/2003 e aceito em 6/4/2004.
} 
formations $\left(\mathrm{S}-\mathrm{F}_{1}, \mathrm{~S}-\mathrm{F}_{2}\right.$ e $\left.\mathrm{S}-\mathrm{F}_{3}\right) . \mathrm{S}-\mathrm{F}_{1}$ was characterized by a higher influence of flooding and the ground water table was closer to the surface; $\mathrm{S}-\mathrm{F}_{2}$ occurred in the central part of the fragment, but presented a strong influence of flooding provided by inundation; and $\mathrm{S}-\mathrm{F}_{3}$, also in the central part of the fragment, occurred the greatest influence from the ground water table. The indicator species of the three forest sub formations were Sebastiania commersoniana and Eugenia uniflora $\left(\mathrm{S}-\mathrm{F}_{1}\right)$; Gymnanthes concolor, Cupania vernalis and Seguieria aculeata $\left(\mathrm{S}-\mathrm{F}_{2}\right)$; and Casearia sylvestris and Allophylus edulis $\left(\mathrm{S}-\mathrm{F}_{3}\right)$. Therefore, in preservation projects, conservation and re-establishment of these ecosystems, the forest community cannot be treated as riparian only, but environmental and, consequently, floristics variations should be considered.

Key words: riparian forest; forest fragment; cluster analysis; sub formations.

\section{INTRODUÇÃO}

A fragmentação e redução das florestas ripárias no Brasil, nos últimos anos, têm aumentado a erosão e causado prejuízo aos mananciais hídricos, reduzindo a biodiversidade (Barbosa, 2000). A Região Sul do País se apresenta apta para a produção agropecuária. Conforme Ab'Saber (2000), tais atividades têm eliminado as matas ripárias no Rio Grande do Sul, sugerindo a necessidade de priorizar sua preservação. Por outro lado, a agricultura e pecuária são de real importância na economia do Estado e, por isso, qualquer medida a ser tomada deve também observar esse aspecto.

Apesar da importância das florestas ripárias, que servem como anteparo, proteção à erosão, filtragem dos sedimentos e produtos químicos utilizados nas lavouras, são realizados poucos estudos relacionados a essas formações florestais na região. A maioria dos estudos sobre a floresta ripária no Brasil é realizada nas regiões sudeste e centro-oeste onde o tema recebeu destaque, sobretudo desde o final da década de 1980. Nestes, é evidenciado que a estrutura e composição florística das florestas ripárias possuem características próprias, relacionadas à região de ocorrência e, apesar da fisionomia aparentemente homogênea, podem também apresentar drásticas variações no ambiente físico e na distribuição de espécies a curta distância.

As variações ambientais agem como fator de seleção natural, agrupando espécies que possuem características próprias (floração, frutificação, dispersão, germinação) e estabelecimento em função da variação estrutural longitudinal e transversal da floresta em relação ao curso do rio (Schiavini, 1992; Walter 1995).

O conhecimento, ainda incipiente, dos ecossistemas ripários no Rio Grande do Sul, associado à necessidade de preservação dessas faixas de vegetação, requer informações ecológicas básicas passíveis de serem utilizadas em processos de preservação, conservação e restabelecimento. Contudo, neste estudo, procurou-se verificar a ocorrência de subformações (agrupamentos) em fragmento de Floresta Estacional Decidual ripária, partindo da análise de espécies diferenciais o que indicará aspectos ambientais associados à vegetação predominante e espécies mais adaptadas a cada condição.

\section{MATERIAL E MÉTODOS}

\section{Localização}

O estudo foi realizado em um fragmento de Floresta Estacional Decidual Aluvial (IBGE, 1992), nas margens do rio Jacuí (3004'36”'S; 52 53’09”W), no sudoeste do município de Cachoeira do Sul, RS. A área estudada é de, aproximadamente, 4 hectares, ocupando $600 \mathrm{~m}$ da margem do rio e largura variada, com máxima de 190 m. A área, pertencente à Cerâmica Kipper, foi escolhida considerando seu estado de conservação, pois, apesar de já ter sido alterada no passado, não apresenta características de intervenção intensas, especialmente no sentido margem-borda.

A oeste da floresta ripária existe cerca de $150 \mathrm{~m}$ de margem com floresta remanescente de Eucalyptus sp. misturado a espécies nativas, a qual é seguida de um trecho de área desprovida de vegetação. A leste (em direção à cidade), a floresta que segue a área de estudo é estreita (máximo de 30 a 40 m), por aproximadamente $200 \mathrm{~m}$, sendo seguida por área alterada e logo adiante interceptada pela ponte do Fandango e cidade. 
O fragmento ocorre numa curva interna do rio, estando sujeito a maior impacto durante as inundações, em períodos de maior intensidade de chuva.

\section{Caracterização geral da área}

A área de estudo pertence ao domínio geológico da Cobertura de Sedimentos Cenozóicos (Kaul, 1990). Moser (1990) classificou o solo como Planossolo, típico de áreas com excesso de água em período temporário ou permanente. Esses solos apresentam horizonte superficial eluvial de textura arenosa ou média, em grande contraste com o horizonte B. A potencialidade agrícola é restrita por causa da textura arenosa ou média e excesso sazonal de água.

Conforme classificação de Köppen, o clima na região pertence à variedade específica "Cfa", definida por apresentar temperatura média do mês mais frio entre $-3^{\circ}$ e $18^{\circ} \mathrm{C}$ e, do mês mais quente superior a $22^{\circ} \mathrm{C}$. A precipitação média anual, distribuída durante todo o ano, está entre 1.500 e $1.600 \mathrm{~mm}$ (Moreno, 1961).

A vegetação pertence à região fitogeográfica de Floresta Estacional Decidual (IBGE, 1992), caracterizada por apresentar duas estações térmicas distintas que provocam a estacionalidade dos elementos arbóreos dominantes do estrato emergente. A queda de temperatura, no inverno, causa a seca fisiológica, propiciando a perda de folhas por $50 \%$ dos indivíduos do dossel (Klein, 1984).

\section{Caracterização do fragmento}

Conforme observações e medições de fatores ambientais na área, constatou-se que a topografia no fragmento é acidentada, tanto no sentido longitudinal como perpendicular ao curso do rio. Em $10 \mathrm{~m}$ do leito do rio ocorre a ribanceira, enquanto as parcelas posteriores apresentam maior altitude, declinando novamente no final da mata (borda). A oeste do fragmento, há formação de canais pelo escoamento da água, no período das enchentes e no leste há irregularidade no terreno formando concavidades isoladas.

Medições mensais analisadas na área juntamente com os dados da PORTOBRAS (Empresa de Portos do Brasil) e cotas altimétricas mostraram que o nível do lençol freático varia no tempo e espaço. Em períodos de enchente e extravasamento do rio, toda ou grande parte da superfície do solo da mata é coberta por água, durante alguns dias, geralmente, em época próxima do inverno (maio a outubro).

Conforme a intensidade das chuvas, a porção central da mata não é inundada, formando uma ilha. Entretanto, em alguns pontos com concavidades isoladas há o acúmulo de água por curto período (10 a 15 dias), de onde a saída está mais influenciada pela infiltração do que pelo escoamento superficial, enquanto em outros, também no meio da mata, formam-se canais onde o escoamento superficial atua mais efetivamente. A borda (final da mata) permanece mais tempo influenciada pela saturação hídrica, pois é adjacente a uma concavidade no terreno (externa à mata), que se mantém com água pela maior parte do ano, formando um pequeno lago, e a margem do rio é afetada durante as enchentes.

A caracterização do solo (20 cm superficais) mostrou que a fração areia foi maior nos primeiros $20 \mathrm{~m}$ da margem, enquanto na borda o solo apresentou maior porcentagem de argila (mais de 25\%), observando-se resultado inverso no teor de matéria orgânica.

\section{Metodologia de análise}

O método de transecto (faixa) é ideal e eficiente quando se deseja estudar gradiente ecológico (Brower e Zar, 1984). Assim, considerando o objetivo de verificar a variação da vegetação dentro do fragmento e a eventual ocorrência de grupos, foram locadas faixas perpendiculares ao rio, distribuídas sistematicamente em intervalos de $50 \mathrm{~m}$ entre si, na margem do rio. Essas faixas foram subdivididas em subunidades amostrais de $10 \mathrm{~m}$ x $10 \mathrm{~m}$ (Figura 1). O fato de o fragmento estudado estar localizado numa curva interna do rio ocasionou a interceptação entre faixas, e, assim, apenas três faixas atravessaram todo o fragmento.

$\mathrm{Na}$ área amostral, a vegetação estudada foi representada por indivíduos arbóreos e arbustivos com circunferência a $1,3 \mathrm{~m}$ do solo $\geq 15 \mathrm{~cm}$ os quais foram identificados e medidos. Lianas tiveram apenas a 
circunferência medida, não sendo identificadas quanto à espécie.

Conforme Braun-Blanquet (1979), a área mínima que representa uma comunidade deve corresponder a um número mínimo de espécies e pode ser obtida pela curva espécie-área. Neste estudo, utilizou-se o número médio de espécies em função da área, plotado em eixos coordenados (McCune e Mefford, 1997).

A determinação de subformações florestais (agrupamentos) no fragmento foi realizada pelo programa TWINSPAN o qual verificou a ocorrência de padrões na distribuição espacial de espécies, associados às condições ambientais locais constatadas no campo (Hill, 1979).

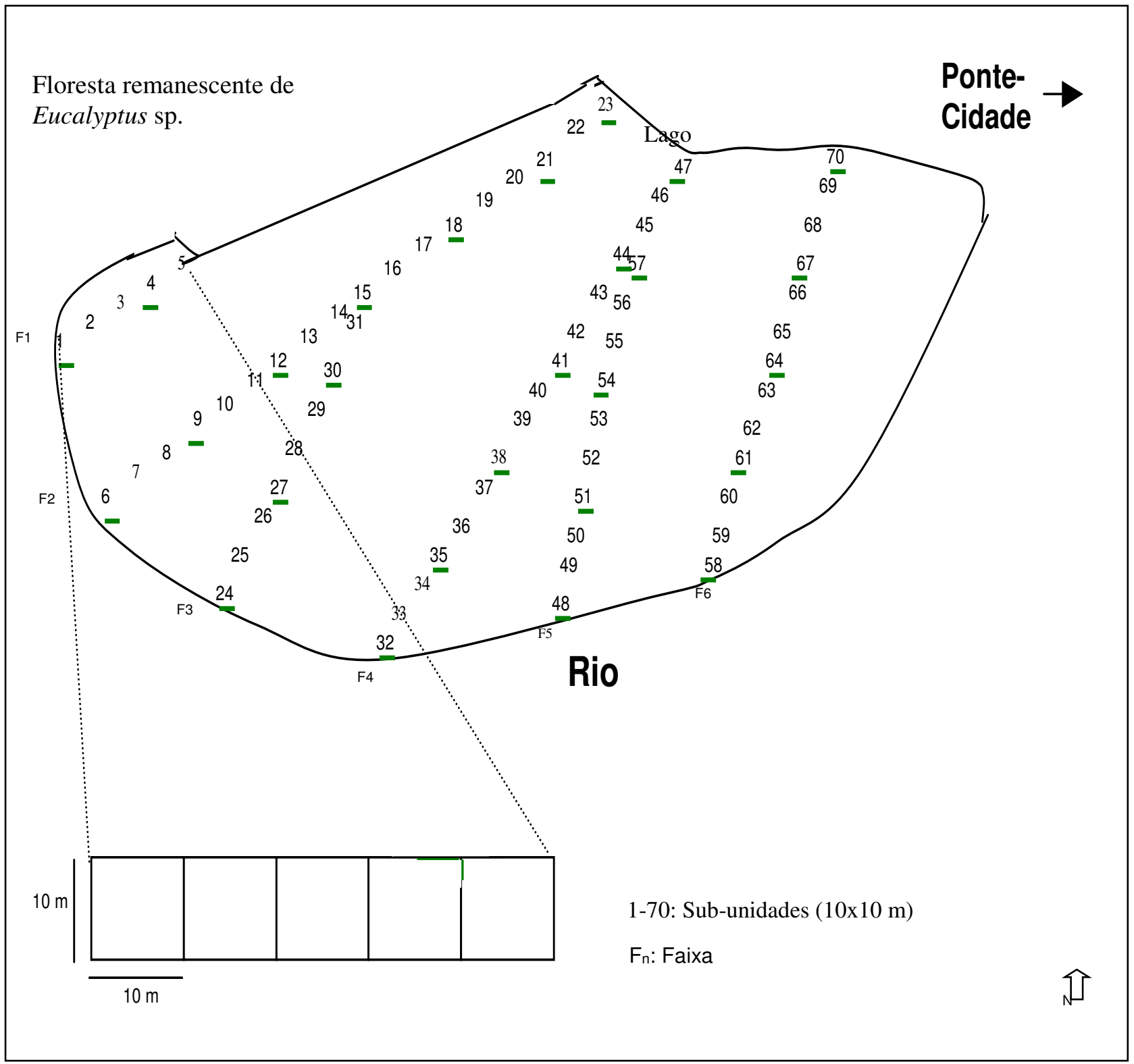

FIGURA 1: Amostragem utilizada no inventário de árvores, arbustos e lianas de um fragmento de Floresta Estacional Decidual Aluvial, Baixo Rio Jacuí, Cachoeira do Sul, RS, Brasil.

FIGURE 1: Sample units used to survey the trees, shrubs and lianas of a fragment of Seasonal Deciduous Alluvial Forest, low Jacuí River, Cachoeira do Sul, RS, Brazil .

$\mathrm{Na}$ análise multivariada, utilizou-se uma matriz de $70 \times 42$, considerando as 70 unidades amostrais (linhas), 40 colunas de espécies, mais uma de lianas e outra de indivíduos mortos. Os dados considerados 
foram de abundância, descartando-se as espécies com menos de três indivíduos as quais foram denominadas raras. Esse procedimento foi baseado na sugestão de Gauch (1982) que considera que as espécies com número reduzido de indivíduos apresentam pouca ou nenhuma influência sobre os resultados dos agrupamentos.

A análise de agrupamento, baseada no conceito de reunir pontos que representam elementos (amostras ou espécies) com características similares, é realizada com base em cálculos matemáticos. $\mathrm{Na}$ fitossociologia, pode ser utilizado o conceito de espécies diferenciais as quais são responsáveis pela divisão de um conjunto de dados, em grupos menores de maior similaridades. Na idéia inicial, estas apresentavam apenas caráter qualitativo, utilizando a presença e ausência de espécies em amostras e desprezando o aspecto de densidade (Kent e Coker, 1992). As espécies diferenciais apresentam preferências ecológicas, e sua presença pode indicar condições ambientais particulares (Hill, 1979).

Hill et al. (1975) desenvolveram um método denominado "Análise de espécies indicadoras" como modo de conciliar variáveis qualitativas (espécies) e quantitativas (densidade), utilizando, para isso, as "pseudoespécies". Com base nesse método, o TWINSPAN (TWo-way INdicator SPecies ANalysis) foi desenvolvido por Hill (1979), como forma de suprir necessidades de ecologistas e fisiologistas cujos dados são conjunto de espécies em amostras definidas. O TWINSPAN utiliza um procedimento multivariado que classifica as unidades em grupos relativamente homogêneos, sendo amplamente aplicado na análise numérica em vegetação e, recentemente, em muitas análises fitossociológicas (Bruce, 1994). O método é hierárquico e divisivo (Hill, 1979), e sua idéia principal está implícita nas pseudoespécies as quais reduzem os dados quantitativos a dados de ausência e presença sem perda de informações. Isso foi possível convertendo os dados de densidade em classes que representam intervalos do número de indivíduos da espécie, ou seja, uma determinada espécie pode ser representada, arbitrariamente, por cinco pseudoespécies, dependendo da variação do número de indivíduos que existe em cada unidade amostral (Hill, 1979).

Para execução da análise, os pontos de corte estipulados para as pseudoespécies foram $0,2,5,10 \mathrm{e}$ 20. De forma mais clara, isso pode ser explicado com o seguinte exemplo: as pseudoespécies são assim denominadas porque apenas uma espécie se divide em uma "série de espécies" (Pseudoespécies 1, 2, 3, 4 e 5). No caso do nível de corte $0,2,5,10,20$, utilizado neste estudo, a pseudoespécie 1 apresenta 1 indivíduo na parcelas; a pseudoespécie 2 tem 2 a 4 e, assim, sucessivamente, até pseudoespécie 5 que corresponde a 20 ou mais indivíduos na parcela.

Basicamente, o método ordena as amostras num eixo, em que é determinado o centróide (centro de gravidade da ordenação) e então é feita uma dicotomização em dois grupos (parcelas ou espécies de um lado e outro do centróide), os quais formam quatro, que, por sua vez, formam oito grupos e, assim, sucessivamente. A cada divisão produzida, é calculado um autovalor (eigenvalue) que indica o quanto da variação dos dados da comunidade foi explicada no eixo de ordenação (McCune e Mefford, 1997). Conforme Gauch (1982), em dados obtidos na natureza, um autovalor em torno de 0,30 é comumente encontrado e utilizado para explicar tais variações. As amostras são classificadas e posteriormente, de forma semelhante, a classificação é feita para espécies utilizando as amostras como base (Hill et al., 1975; Silva, 2002).

Para definição dos agrupamentos, além da análise multivariada, foram consideradas as características observadas no campo e possíveis interpretações ecológicas para a formação de tais grupos. Conforme Kent e Coker (1992), o número de subdivisões depende do tamanho e característica do conjunto de dados, considerando que nem todas as divisões são definidas como um grupo, mas aquelas que podem ser verificadas in loco e explicadas a posteriori. Se a divisão produzir subgrupos que não refletem as características da floresta, estes devem, então, ser mesclados.

\section{RESULTADOS E DISCUSSÃO}

\section{Composição Florística}

A amostragem pode ser considerada suficiente para caracterizar a vegetação. Conforme se observa na Figura 2, houve uma razoável tendência à estabilização. Em florestas naturais, a tendência à estabilização é suficiente para representar a vegetação (Jardim e Hosokawa, 1986; Araujo, 1998; Longhi et al., 1999a). 
A família mais representativa na área estudada foi Myrtaceae, com 11 espécies, seguida de Rubiaceae (quatro espécies), Euphorbiaceae, Fabaceae, Flacourtiaceae, Mimosaceae, Sapindaceae e Sapotaceae (três espécies), Boraginaceae (duas espécies) e 14 famílias com uma espécie apenas (Tabela 1). A família Myrtaceae está sempre presente com grande representatividade em ecossistemas ripários, conforme pode ser observado nos trabalhos realizados por Tabarelli et al. (1992), Kilka (2002) e Longhi et al. (2001).

A composição florística da floresta ripária estudada (Tabela 1) é semelhante a de outros fragmentos de vegetação na mesma região fitogeográfica, mas sem a interferência dos rios (Bearzi et al., 1992; Longhi et al., 1999a; Vaccaro et al., 1999). Rodrigues e Nave (2000), considerando as condições bastante específicas das florestas ripárias, comentaram que a maior diferença entre essa vegetação e as formações não-ripárias está relacionada com os aspectos quantitativos e fisionômicos.

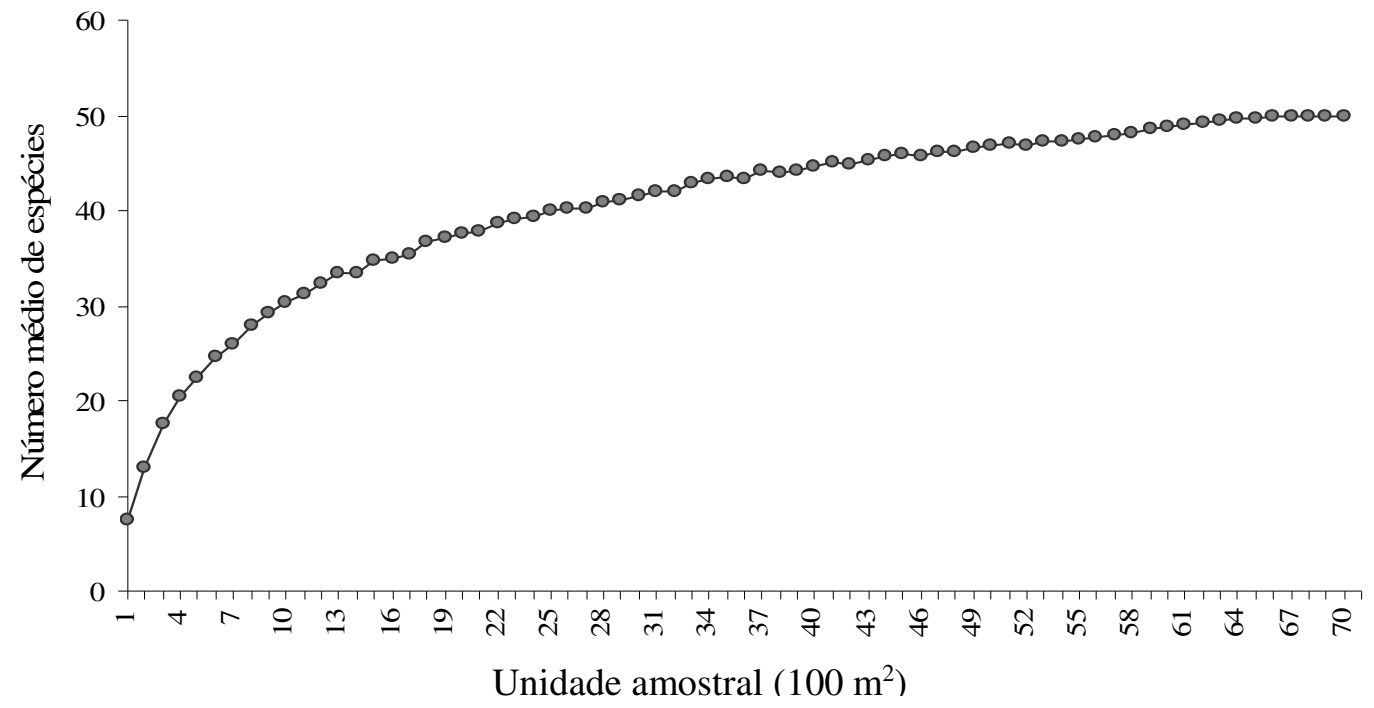

FIGURA 2: Curva espécie-área, considerando indivíduos com CAP $\geq 15 \mathrm{~cm}$, em Floresta Estacional Decidual Aluvial, no Baixo Rio Jacuí, Cachoeira do Sul, RS, Brasil.

FIGURE 2: Species-area curve, considering individuals with $\mathrm{GBH} \geq 15 \mathrm{~cm}$, in Seasonal Deciduous Alluvial Forest, in the low River Jacuí, Cachoeira do Sul, RS, Brazil.

TABELA 1: Espécies encontradas em fragmento de Floresta Estacional Decidual Aluvial, Baixo Rio Jacuí, Cachoeira do Sul, RS, Brasil.

TABLE 1: Species found in the fragment of Seasonal Deciduous Alluvial Forest, low Jacuí River, Cachoeira do Sul, RS, Brazil.

\begin{tabular}{lll}
\hline \multicolumn{1}{c|}{ Família } & \multicolumn{1}{c}{ Espécie } & \multicolumn{1}{c}{ Nome Vulgar } \\
\hline Annonaceae & Rollinia salicifolia Schltdl. & araticum-salso \\
Boraginaceae & Cordia ecalyculata Vell. & louro-mole \\
Boraginaceae & Patagonula americana L. & guajuvira \\
Caesalpiniaceae & Apuleia leiocarpa (Vogel) J.F.Macbr. & grápia \\
Ebenaceae & Diospyros inconstans Jacq. & maria-preta \\
Erythroxylaceae & Erythroxylum deciduum A.St.-Hil. & cocão \\
Euphorbiaceae & Gymnanthes concolor Spreng. & laranjeira-do-mato \\
Euphorbiaceae & Sebastiania brasiliensis Spreng. & branquilho-leiteiro \\
Euphorbiaceae & Sebastiania commersoniana (Baill.) L.B.Sm. et Downs & branquilho \\
Fabaceae & Lonchocarpus nitidus (Vogel) Benth. & farinha-seca \\
Fabaceae & Machaerium paraguariense Hassl. & canela-do-brejo \\
\hline
\end{tabular}


TABELA 1: Continuação ...

TABLE 1: Continued ...

\begin{tabular}{|c|c|c|}
\hline Família & Espécie & Nome Vulgar \\
\hline Fabaceae & Myrocarpus frondosus M.Allemão & cabriúva \\
\hline Flacourtiaceae & Banara tomentosa Clos & guaçatunga-branca \\
\hline Flacourtiaceae & Casearia decandra Jacq. & guaçatunga \\
\hline Flacourtiaceae & Casearia sylvestris $\mathrm{Sw}$. & carvalinho \\
\hline Lauraceae & Ocotea pulchella Mart. & canela-lageana \\
\hline Loganiaceae & Strychnos brasiliensis (Spreng.) Mart. & anzol-de-lontra \\
\hline Meliaceae & Trichilia elegans A.Juss. & pau-ervilha \\
\hline Mimosaceae & Inga vera Willd. & ingá-beira-de-rio \\
\hline Mimosaceae & Mimosa bimucronata (DC.) Kuntze & maricá \\
\hline Mimosaceae & Parapiptadenia rigida (Benth.) Brenan & angico-vermelho \\
\hline Moraceae & Sorocea bonplandii (Baill.) W.C.Burger et al. & cincho \\
\hline Myrtaceae & Blepharocalyx salicifolius (Kunth) O.Berg & murta \\
\hline Myrtaceae & Calyptranthes concinna DC. & guamirim-de-facho \\
\hline Myrtaceae & Campomanesia xanthocarpa O.Berg & guabiroba \\
\hline Myrtaceae & Eugenia hyemalis Cambess. & guamirim-folha-miúda \\
\hline Myrtaceae & Eugenia involucrata DC. & cerejeira-do-mato \\
\hline Myrtaceae & Eugenia mansoi Berg & mirtácea \\
\hline Myrtaceae & Eugenia ramboi D.Legrand & batinga-branca \\
\hline Myrtaceae & Eugenia uniflora $\mathrm{L}$. & pitanga \\
\hline Myrtaceae & Eugenia uruguayensis Cambess. & batinga-vermelha \\
\hline Myrtaceae & Myrcianthes pungens (O.Berg) D.Legrand & guabijú \\
\hline Myrtaceae & Myrciaria tenella (DC.) O.Berg & cambuim \\
\hline Phytolaccaceae & Seguieria aculeata L. & cipó-umbú \\
\hline Polygonaceae & Ruprechtia laxiflora Meisn. & marmeleiro-do-mato \\
\hline Rhamnaceae & Scutia buxifolia Reissek & coronilha \\
\hline Rubiaceae & Chomelia obtusa Cham. et Schltdl. & viuvinha \\
\hline Rubiaceae & Guettarda uruguensis Cham. et Schltdl. & veludinho \\
\hline Rubiaceae & Randia armata (Sw.) DC. & limoeiro-do-mato \\
\hline Rubiaceae & Terminalia australis Cambess. & amarilho; sarandi \\
\hline Sapindaceae & Allophylus edulis (A.St.-Hil. et al.) Radlk. & chal-chal \\
\hline Sapindaceae & Cupania vernalis Cambess. & camboatá-vermelho \\
\hline Sapindaceae & Matayba elaeagnoides Radlk. & camboatá-branco \\
\hline Sapotaceae & Chrysophyllum gonocarpum (Mart. \& Eichler) Engl. & aguaí-da-serra \\
\hline Sapotaceae & Chrysophyllum marginatum (Hook. et Arn.) Radlk. & aguaí-leiteiro \\
\hline Sapotaceae & Pouteria gardneriana (DC.) Radlk. & mata-olho \\
\hline Tiliaceae & Luehea divaricata Mart. et Zucc. & açoita-cavalo \\
\hline Ulmaceae & Celtis iguanaea (Jacq.) Sargent & esporão-de-galo \\
\hline Verbenaceae & Vitex megapotamica (Spreng.) Moldenke & tarumã \\
\hline
\end{tabular}

Comparando as espécies encontradas neste estudo, no baixo Rio Jacuí, com o trabalho de Daniel (1991) no Rio dos Sinos, Tabarelli et al. (1992) no Ibicuí e Longhi et al. (1999b) no Ibicuí-Mirim constatou-se que a similaridade foi maior com as áreas de estudo mais próximas.

As espécies mais comumente encontradas na região fitogeográfica por Silva et al. (1992), Nascimento et al. (2000) e Araujo et al. (2000), também observadas na floresta ripária, são Allophylus edulis, Luehea divaricata, Cupania vernalis, Matayba elaeagnoides, Myrocarpus frondosus, Casearia sylvestris, Trichilia elegans e Gymnanthes concolor. A composição florística do fragmento estudado 
apresentou espécies higrófitas deciduais, adaptadas ao ambiente aluvial da região, como Luehea divaricata, Vitex megapotamica, Inga vera, Ruprechtia laxiflora e Sebastiania commersoniana (IBGE, 1992).

\section{Análise de agrupamentos}

Tomando por base o estudo de gradiente da vegetação, a análise de Cluster definiu três subformações claramente distintas no fragmento estudado as quais se diferenciaram por apresentar variação longitudinal e transversal em relação ao leito do rio (Figura 3). A classificação das unidades amostrais em grupos ocorreu da seguinte forma: a primeira divisão, com autovalor de 0,407, mostrou que existe similaridade na vegetação das unidades amostrais do dique e borda (SubFormação 1: $S-F_{1}$ ) e diferença daquelas que ocorreram no meio do fragmento o que indicou a existência de espécies com diferentes graus de tolerância à saturação hídrica.

Sebastiania commersoniana e Eugenia uniflora foram espécies indicadoras da S-F $\mathrm{F}_{1}$ (dique e borda), conforme pode-se observar na Figura 4, sendo descritas por Reitz et al. (1983) e Lorenzi (1998) como adaptadas às condições ambientais de floresta ripária por serem higrófilas e heliófilas, enquanto Casearia sylvestris e lianas foram indicadoras do agrupamento do meio do fragmento.

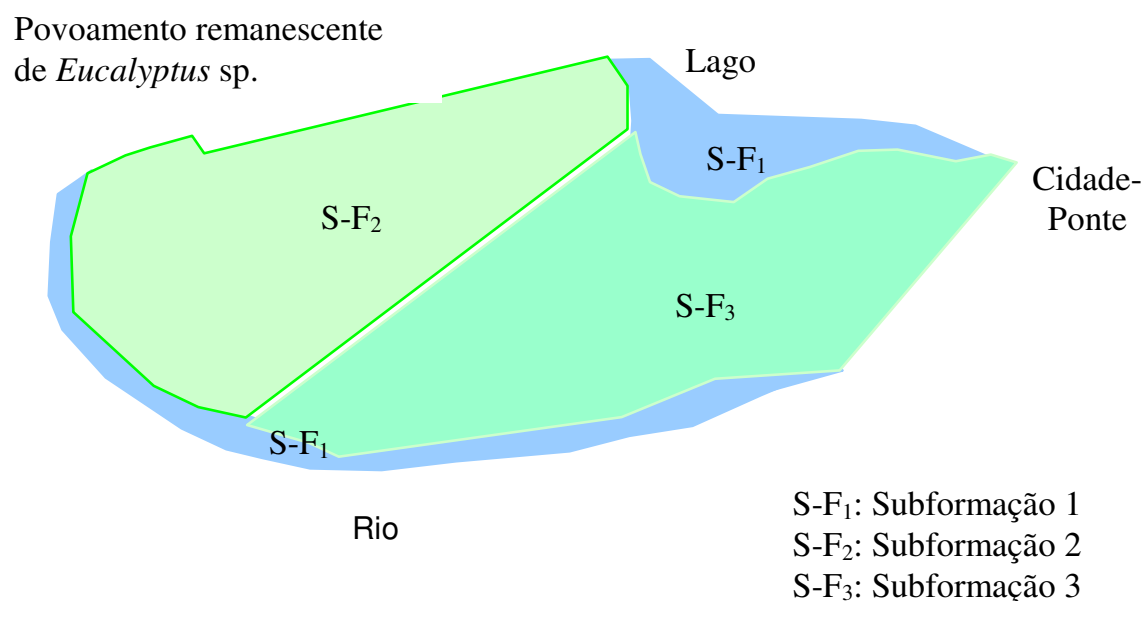

FIGURA 3: Subformações florestais em fragmento de Floresta Estacional Decidual Alluvial, Baixo Rio Jacuí, Cachoeira do Sul, RS, Brasil.

FIGURE 3: Forest Subformation in the fragment of Seasonal Deciduous Alluvial Forest fragment, in the low River Jacuí, Cachoeira do Sul, RS, Brazil.

A segunda divisão, com autovalor de 0,301, separou as amostras do meio da mata em duas subformações, uma a oeste $\left(\mathrm{S}-\mathrm{F}_{2}\right)$ e outra a leste $\left(\mathrm{S}-\mathrm{F}_{3}\right)$ (Figura 3). Analisando os resultados matemáticos, dados complementares e relatórios de campo, foi possível observar que, no lado oeste, a interferência do rio é maior no período de enchentes. Isso pode ser explicado pelo fluxo do rio, no sentido oeste-leste, que encontra como barreira o fragmento em curva, sendo, nesse local, formados os canais, enquanto a leste ocorre acúmulo d'água em alguns pontos por maior tempo do que na $\mathrm{S}-\mathrm{F}_{2}$, considerando a menor vazão. A formação de canais já foi observada em floresta ripária no Baixo Rio Piratini, sendo descrito por Kilka (2002) como "canais semiativos", em razão de ocorrerem somente após as enchentes.

A subformação $2\left(\mathrm{~S}_{-} \mathrm{F}_{2}\right)$ foi indicada pelas espécies Cupania vernalis, Seguieria aculeata e Gymnanthes concolor (Figura 4). As duas primeiras espécies são heliófilas (Reitz et al., 1983 e Marchiori, 2000), ocorrendo no ambiente, provavelmente, por causa das perturbações periódicas causadas pelo extravasamento do rio, enquanto Gymnanthes concolor é comum no sub-bosque da floresta, compondo o estrato inferior (Longhi et al., 2000; Nascimento et al., 2000) e observada por Vaccaro et al. (1999) em estágios mais avançados de sucessão. 


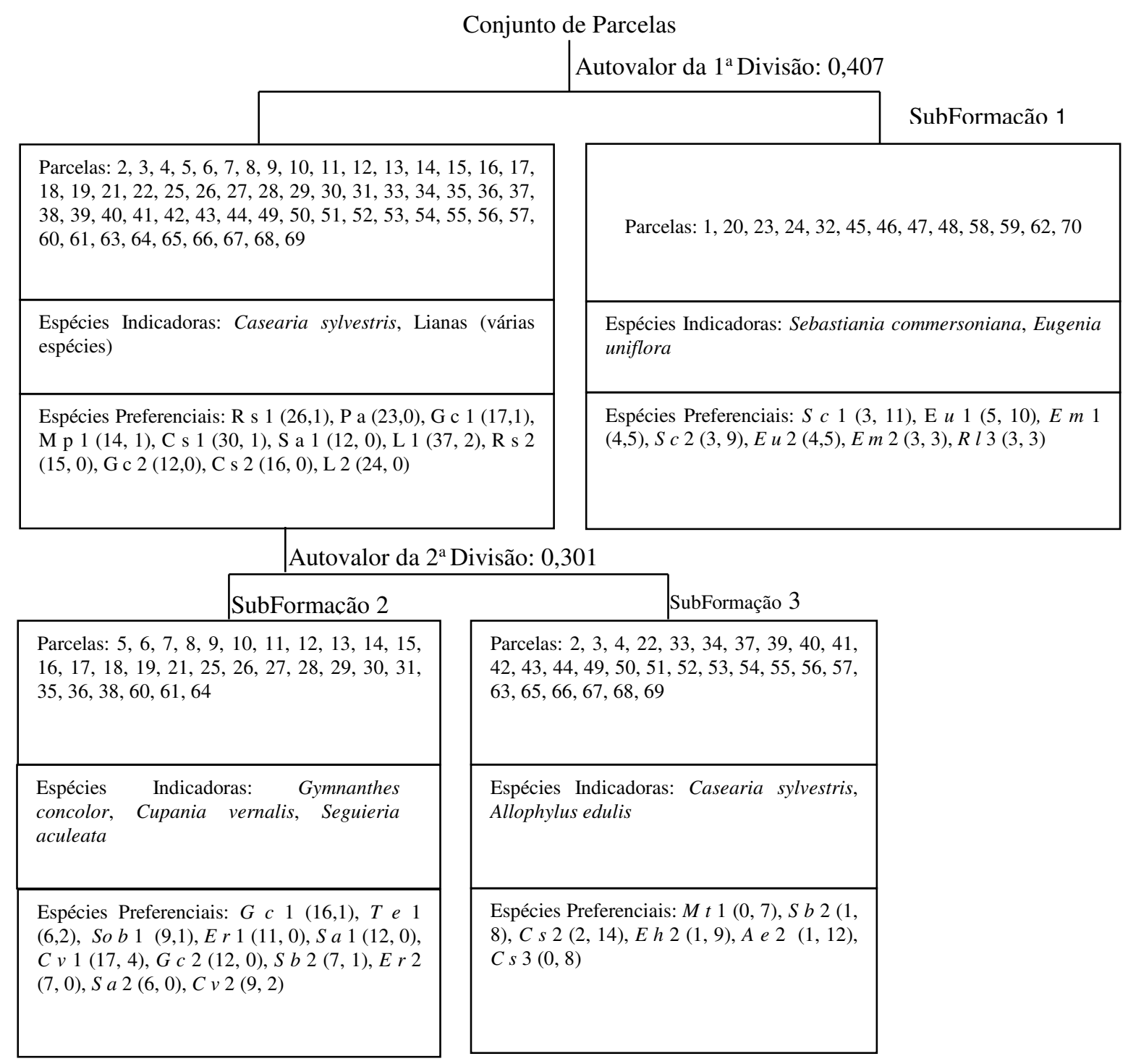

Em que: $R s$ (Rollinia salicifolia), $P$ a (Patagonula americana), $G c$ (Gymnanthes concolor), $M p$ (Machaerium paragrariense), C $s$ (Casearia sylvestris), L (Lianas), $S c$ (Sebastiania commersoniana), E u (Eugenia uruguayensis), E $m$ (Eugenia mansoi), $R l$ (Ruprechtia laxiflora), $T$ e (Trichilia elegans), $S b$ (Sebastiania brasiliensis), E $r$ (Eugenia ramboi), $S$ a (Seguieria aculeata), $C v$ (Cupania vernalis), So $b$ (Sorocea bonplandii), Mt (Myrciaria tenella), E h (Eugenia hyemalis), A e (Allophylus edulis).

Observação: O número depois da abreviatura se refere a pseudoespécie (nível de corte).

$\mathrm{O}$ número entre parênteses se refere às parcelas em que estão contidas as pseudoespécies, em ambos os lados da divisão.

FIGURA 4: Classificação das unidades amostrais em três grupos, caracterizando as subformações internas em fragmento de Floresta Estacional Decidual Aluvial, no Baixo Rio Jacuí, Cachoeira do Sul, RS, Brasil.

FIGURE 4: Classification of the samples units in to three groups, characterizing the subformations of the fragmento Seasonal Deciduous Alluvial Forest, in the low Jacuí River, Cachoeira do Sul, RS, Brazil.

As espécies indicadoras da subformação $\mathrm{S}^{-\mathrm{F}_{3}}$, Casearia sylvestris e Allophylus edulis são comuns em matas ripárias. A primeira, apesar de ter melhor performance sob condição de luminosidade, ocorre também em florestas mais densas; enquanto Allophylus edulis é espécie esciófila (Reitz et al., 1983; 
Lorenzi, 1998). Esse fato pode indicar a situação de estágio com menor alteração da subformação $S-\mathrm{F}_{3}$, conseqüentemente, possibilitando a ocorrência e freqüência de espécies adaptadas aos ambientes sombreados. Por outro lado, a permanência de água por maior tempo na área pode ter influenciado na presença das espécies, considerando que as parcelas 3 e 4 (cotas baixas) da faixa 1 (Figura 3), permaneceram com água por maior período, ficaram classificadas na $\mathrm{S}-\mathrm{F}_{3}$ e apresentaram Allophylus edulis e Casearia sylvestris.

As espécies preferenciais (abreviadas) na Figura 4 que também definem as subformações mostram o número de parcelas em que ocorreu a pseudoespécie. No caso da subformação 1, que apresentou Eugenia mansoi 2 e Ruprechtia laxiflora 3 em três parcelas nos dois lados da divisão, e mesmo assim foram preferenciais na $\mathrm{S}-\mathrm{F}_{1}$, ocorreu em conseqüência desse grupo ter menos parcelas.

A partir das subformações, foram gerados grupos de espécies, provavelmente, com semelhantes preferências ambientais, em que seis grupos foram considerados válidos. No entanto, o conhecimento limitado da auto-ecologia de espécies nativas, restringe uma discussão mais aprofundada.

O que foi possível distinguir é que a $1^{\text {a }}$ divisão (autovalor 0,606 ) apresentou grupos $(\mathrm{G})$ de espécies, sobretudo, restritas à porção central do fragmento e de borda-dique, ambos com algumas espécies comuns em todos os trechos da mata. Consecutivas divisões dos grupos formaram subgrupos que separavam as espécies mais comuns daquelas restritas a determinada porção: $\mathrm{G}_{1}$, representado por espécies de dique e borda; $\mathrm{G}_{2}$, por espécies com maior número de indivíduos no dique-borda, mas ocorrendo nas outras subformações; $\mathrm{G}_{3}$, formado por espécies que ocorrem em todo o fragmento, mas sobretudo na $\mathrm{S}-\mathrm{F}_{3} ; \mathrm{G}_{4}$, por espécies comuns nas subformações $\mathrm{S}-\mathrm{F}_{2}$ e $\mathrm{S}-\mathrm{F}_{3}$; e $\mathrm{G}_{5}$, por aquelas restritas à $\mathrm{S}-\mathrm{F}_{2}$, além de lianas que apesar de ocorrerem na $S-F_{3}$, apresentam grande número de indivíduos na $S-F_{2}$. A subdivisão do $G_{3}$ não ficou claramente definida.

Contudo, foi possível diferenciar as subformações da seguinte forma:

A S-F F $_{1}$ (sitio 1) foi caracterizada, sobretudo, pela presença conjunta de Sebastiania commersoniana e Eugenia uniflora. Essas espécies, além de Eugenia mansoi, são mais freqüentes e não-exclusivas da subformação, porém nota-se serem adaptadas à saturação hídrica, pois foram mais representativas na $\mathrm{S}-\mathrm{F}_{3}$ do que na S-F $\mathrm{F}_{2}$. Como espécies exclusivas dessa subformação estão Inga vera, Calyptranthes concinna e Terminalia australis que ocorreram somente na margem. Estas, apesar da baixa frequiência, apresentaram densidade relativamente alta o que caracteriza o padrão de distribuição espacial agregado.

A Seguieria aculeata foi espécie exclusiva da $\mathrm{S}-\mathrm{F}_{2}$. Além desta espécie, $\mathrm{S}-\mathrm{F}_{2}$ e $\mathrm{S}-\mathrm{F}_{1}$ foram diferenciadas pela elevada frequiência de Ocotea pulchella, Sorocea bonplandii, Randia armata, Rollinia salicifolia, Trichillia elegans, Patagonula americana e Casearia sylvestris na $\mathrm{S}-\mathrm{F}_{2}$, enquanto, quando foram comparados os sítios $\mathrm{S}_{-} \mathrm{F}_{2}$ e $\mathrm{S}-\mathrm{F}_{3}$, se constatou maior freqüência de Eugenia ramboi na sub-formação 2 e de Sebastiania commersoniana na subformação 3.

As espécies exclusivas no sítio 3 foram Cordia ecalyculata, Eugenia uruguayensis e Myrciaria tenella. Comparando-se S- $\mathrm{F}_{3}$ e S-F , observou-se a presença de Patagonula americana e Casearia sylvestris na primeira e ausência na segunda subformação.

A $S-F_{1}$ (parcelas de borda e dique) ocorreu em cotas mais baixas, em relação àquelas centrais, conseqüentemente, as espécies tolerantes ao lençol freático mais próximo da superfície foram favorecidas.

Assim, foi possível constatar que a composição florística do fragmento, ou de suas subformações internas, são determinadas pelos fatores ambientais predominantes em determinado espaço. Conforme Runhaar e Haes (1994), a composição de espécies está em equilíbrio com as condições do ambiente até que estas mudem e, conseqüientemente, as espécies já não serão indicativas daquele ambiente, tendendo a serem substituídas.

Também sob essa perspectiva, confirma-se o fato de que a $S-\mathrm{F}_{2}$ e $\mathrm{S}-\mathrm{F}_{3}$, por ocorrerem no meio da mata, em cotas mais elevadas, sob condição semelhante de solos, além de estarem num mesmo grupo (maior similaridade florística) na primeira divisão, posteriormente, caracterizaram dois subgrupos. Nesse caso, o tipo de interferência, provavelmente, foi determinante nessa classificação. A S-F $F_{2}$ sofreu interferência 
intensa por curto período, enquanto na $\mathrm{S}-\mathrm{F}_{3}$ ocorreu o contrário, o impacto inicial durante as enchentes foi menor, mas as concavidades formadas no terreno se mantinham por maior tempo.

$\mathrm{Na} \mathrm{S}-\mathrm{F}_{2}$, as enchentes tiveram maior impacto, em especial nas unidades próximas aos canais, proporcionando queda de árvores com maior diâmetro, onde se observou que, em razão das copas estarem entrelaçadas por cipós, outras árvores caíam juntas, conseqüentemente, ocorrendo maior entrada de luz. O fato das concavidades, na $S-F_{3}$, estarem distribuídas no meio do terreno e não na forma de canais foi o que, provavelmente, proporcionou a variação ambiental em relação à S-F $\mathrm{F}_{2}$.

Um aspecto que deve ser mencionado é que algumas unidades amostrais como 2,3 e 4, apesar de estarem a oeste $\left(\mathrm{S}-\mathrm{F}_{2}\right)$ ficaram classificadas como características da $\mathrm{S}-\mathrm{F}_{3}$. Na faixa 2, entre as unidades 19 e 23, houve a sequiência das parcelas $20\left(\mathrm{~S}-\mathrm{F}_{1}\right), 21\left(\mathrm{~S}-\mathrm{F}_{2}\right)$ e $22\left(\mathrm{~S}-\mathrm{F}_{3}\right)$ e, na faixa 6 , entre a 58 e 59 (dique ou S-F $\left.F_{1}\right)$ e $65\left(S-F_{3}\right)$ ocorreram a 60 e 61 na S-F, 62 na S-F, 63 na S-F -64 da S-F $F_{2}$ Entre estas, as unidades amostrais $20\left(15^{\text {a }}\right.$ subunidade da faixa 2$)$ e $62\left(5^{\text {a }}\right.$ subunidade da faixa 6$)$ ocorreram nas cotas mais baixas, tendo maior influência da flutuação do lençol freático. As demais parcelas apresentaram "mistura" de características, representando trechos de transição entre subformações.

As subformações apresentaram grande semelhança quanto aos valores de área basal, observando-se 29,6, 30,6 e 29,83 $\mathrm{m}^{2} /$ ha, respectivamente, para $S-F_{1}, S-F_{2}$ e $S-F_{3}$. Da mesma forma ocorreu em relação ao número de indivíduos por hectare na $S-F_{1}(1.764)$ e $F_{2}$ (1.727) que apresentaram maior diferença em relação a $\mathrm{S}_{-} \mathrm{F}_{3}$, em que foram verificados 2.007 indivíduos. Os valores de área basal foram mais baixos do que os encontrados nas florestas de interflúvio, sendo observados 35,28 $\mathrm{m}^{2} /$ ha (Machado e Longhi, 1990), 32,56 $\mathrm{m}^{2} /$ ha (Bearzi et al., 1992) que consideraram apenas os indivíduos com CAP $\geq 30 \mathrm{~cm}$. Em floresta ripária, Durlo et al. (1982), avaliando a mesma população (CAP $\geq 15 \mathrm{~cm})$, observaram valor inferior $\left(22,75 \mathrm{~m}^{2} / \mathrm{ha}\right)$, assim como Kilka (2002), no Baixo Rio Piratini (23,14 e 20,10m²/ha). Sampaio et al. (2000) descreveram que a área com maior influência hídrica apresentou árvores mais finas e adensadas, considerando que a drenagem tem forte interferência sobre a estrutura da comunidade arbórea, explicando assim a maior densidade na S-F $F_{3}$.

A distribuição diamétrica apresentou forma "J invertido" em todas as subformações. Essa curva representa o equilíbrio dinâmico da floresta que está se auto-regenerando, pois a maior proporção dos indivíduos ocorreu nas primeiras classes, diminuindo naquelas de diâmetros maiores (Figura 5).

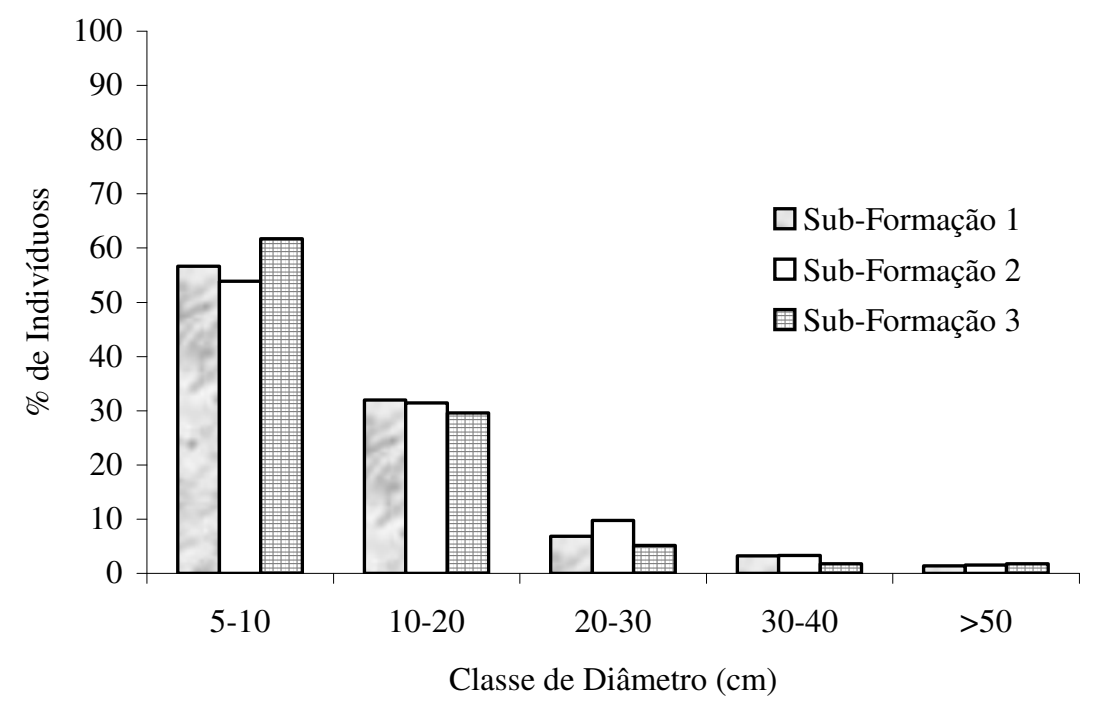

FIGURA 5: Percentagem de indivíduos em classes diamétricas, de três subformações florestais em fragmento de Floresta Estacional Decidual Aluvial, Cachoeira do Sul, RS, Brasil.

FIGURE 5: Individuals' percentage in classes of diameter, of three forest subformations in a fragment of Seasonal Deciduous Alluvia Forest, low Jacuí River, Cachoeira do Sul, RS, Brazil. 
$\mathrm{Na}$ estrutura vertical, observou-se que a maior proporção, em todas as subformações, foi na classe de 5 a $10 \mathrm{~m}$ de altura. Nessa classe, a S-F $\mathrm{F}_{2}$ apresentou menos indivíduos, mas teve mais árvores com altura igual ou maior que $15 \mathrm{~m}$ (Figura 6).

Kilka (2002) também constatou que na floresta ripária, cuja influência do rio é esporádica, as espécies arbóreas se apresentam mais altas e com indivíduos de maiores diâmetros. Apesar da $\mathrm{S}-\mathrm{F}_{2}$ ser influenciada com as enchentes, a interferência é por período curto.

A S-F $F_{1}$ apresentou poucos indivíduos (2) com altura maior que $15 \mathrm{~cm}$, mas esteve proporcionalmente bem representada nas outras classes. Na subformação 3 ocorreu maior proporção de indivíduos entre 5 e $10 \mathrm{~m}$ o que era esperado, considerando que as espécies melhor representadas, Casearia sylvestris e Allophylus edulis, são de baixo a médio porte.

$\mathrm{Na} \mathrm{S}-\mathrm{F}_{1}$, as espécies que ocuparam o estrato superior foram Luehea divaricata e Machaerium paraguariense. Na S- $\mathrm{F}_{2}$, destacaram-se Parapiptadenia rigida, Cupania vernalis, Patagonula americana, Ocotea pulchella, Matayba elaeagnoides, Ruprechtia laxiflora e na $\mathrm{S}_{3} \mathrm{~F}_{3}$, foram Parapiptadenia rigida, Patagonula americana, Matayba elaeagnoides, Luehea divaricata e Ruprechtia laxiflora.

Floristicamente, a floresta parece estar num estágio avançado de sucessão, apresentando espécies características desse estágio sucessional como Ruprechtia laxiflora, Luehea divaricata, Allophylus edulis, Chrysophyllum gonocarpum, Sorocea bonplandii, Apuleia leiocarpa e Eugenia involucrata (Reitz et al., 1983; Longhi, 1995; Marchiori, 2000). Porém, é válido salientar que esse tipo de floresta, mesmo nessa condição, se apresenta num processo bastante dinâmico, pois as mudanças sazonais causam alterações freqüentes, o que sempre as mantêm com presença expressiva de espécies pioneiras como Casearia sylvestris, Ocotea pulchella, Eugenia uniflora, Sebastiania commersoniana e Chrysophyllum marginatum, descritas por Reitz et al. (1983) e Lorenzi (1998).

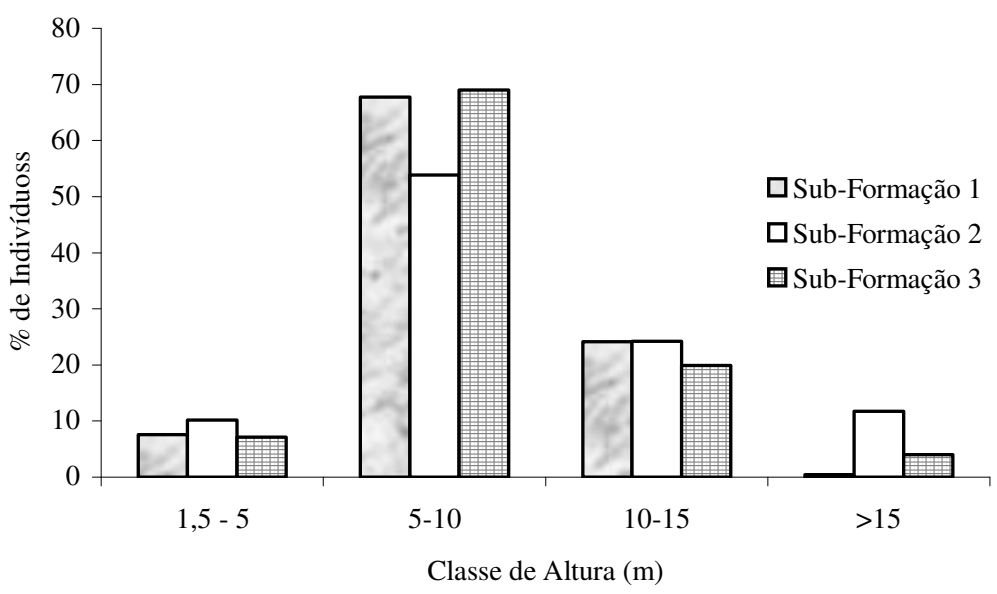

FIGURA 6: Percentagem de indivíduos por classe de altura em três subformações vegetais de fragmento de Floresta Estacional Decidual Ripária, Cachoeira do Sul, RS, Brasil.

FIGURE 6: Individuals' percentage per height class in three vegetation subformations in Seasonal Deciduous Riparian Forest fragment, low River Jacuí, Cachoeira do Sul, RS, Brazil.

Nesse contexto, pode-se descrever que tanto o fragmento, como suas subformações florestais internas se apresentam num estágio sucessional avançado, considerando as características de ambiente ripário.

\section{CONCLUSÕES}

Os resultados permitem concluir que: 
Estruturalmente, a floresta se apresenta com características de florestas em estágio avançado de sucessão, dentro do contexto ripário, considerando que todas as formações encontradas estão se auto-regenerando;

Existem três formações internas no fragmento, associadas às diferentes influências do rio, tanto no sentido transversal como longitudinal. Assim, a vegetação dessas florestas não pode ser tratada unicamente como ripária, mas considerando tais variações.

Sebastiania commersoniana e Eugenia uniflora estão adaptadas à porção onde existe maior impacto e com lençol freático aflorando na maior parte do ano; Gymnanthes concolor e Cupania vernalis são espécies tolerantes a freqüentes alagamentos, mas não à situação de permanência destes, e Allophylus edulis e Casearia sylvestris foram espécies tolerantes a locais com nível do lençol freático mais alto. Essas seis espécies podem ser indicadas no reflorestamento e enriquecimento de áreas com semelhantes características ambientais.

\section{REFERÊNCIAS BIBLIOGRÁFICAS}

AB'SABER, A. N. O suporte geológico das florestas beiradeiras (Ciliares). In: RODRIGUES, R. R.; LEITÃO-FILHO, H. de F. (Eds.) Matas ciliares: conservação e recuperação. São Paulo: Editora da Universidade de São Paulo, 2000. p.15-25.

ARAUJO, M. M. Vegetação e banco de sementes do solo de florestas sucessionais na Região do Baixo Rio Guamá, Benevides, Pará, Brasil. 1998. 86p. Dissertação (Mestrado em Ciências Florestais) - Faculdade de Ciências Agrárias do Pará, Belém, 1998.

ARAUJO, M. M.; LONGHI, S. J.; KELlinG, M. B.; BORSOI, G. A.; HOPPE, J. M.; MÜLLER, I. Padrões de distribuição espacial de espécies florestais em fragmento de floresta estacional decidual, Santa Maria-RS, Brasil. In: SIMPÓSIO LATINO AMERICANO SOBRE MANEJO FLORESTAL, 1., 2000, Santa Maria. Anais... Santa Maria: UFSM - Programa de Pós-graduação em Engenharia Florestal, 2000. p.345-360.

BARBOSA, L. M. Considerações gerais e modelos de recuperação de formações ciliares. In: RODRIGUES, R. R.; LEITÃO-FILHO, H. de F. (Eds.) Matas ciliares: conservação e recuperação. São Paulo: Editora da Universidade de São Paulo, 2000. p.289-312.

BEARZI, R. C.; POLTRONIERI, V. C.; LONGHI, S. J. Estrutura fitossociológica do Morro de Três Marias em Santa Maria - RS. In: CONGRESSO FLORESTAL ESTADUAL, 7., 1992, Nova Prata. Anais... Santa Maria: CEPEF/FATEC - UFSM, 1992. p.376-394.

BRAUN-BLANQUET, J. Fitosociologia: base para el estudio de las comunidades vegetales. 3.ed. Madrid: H. Blume Ediciones, 1979. $820 \mathrm{p}$.

BROWER, J. E.; ZAR, J. H. Field and laboratory methods for general ecology. 2.ed. Iowa: Brown Publishers, 1984. 226p.

BRUCE, R. G. H. The application of quantitative method of classification to strategic ecological survey in Britain. In: KLIJN, F. (Ed.) Ecossistem classification for environmental management. Netherlands: Klwer Academic Publishers, 1994. p.173-182.

DANIEL, A. Estudo fitossociológico arbóreo/arbustivo da mata ripária da Bacia Hidrográfica do Rio dos Sinos, RS. Pesquisa (Botânica), n. 42, p.15-199, 1991.

DURLO, M. A.; MARCHIORI, J. N. C.; LONGHI, S. J. A composição e estrutura da mata secundária no vale do rio Jacuí, RS. Ciência e Natura, n.4, p.129-139, 1982.

GAUCH, H. G. Multivariate analysis in community ecology. Cambridge: Cambridge University Press, 1982. 180p.

HILL, M. O. TWINSPAN: a FORTRAN program fo arranging multivariate data in an ordered two way table by classification of individual and attributes. Ithaca, NY: Cornell University, 1979. 60p.

HILL, M. O.; BUNCE, R. G. H.; SHAW, M. W. Indicator species analysis, a divisive polythetic method of classification, and its application to a survey of native pinewoods in Scotland. The Journal of Ecology, v. 63, n.2, p.597-613, 1975.

IBGE. Manual técnico da vegetação brasileira. Rio de Janeiro, 1992. 92p.

JARDIM, F. C. S.; HOSOKAWA, R. T. Estrutura da floresta equatorial úmida da Estação Experimental de Silvicultura Tropical do INPA. Acta Amazônica, n.16/17, p.411-508, 1986. 
KAUL, P. F. T. Geologia. In: IBGE. Geografia do Brasil: Região Sul. Rio de Janeiro, 1990. p. $29-54$.

KENT, M.; COKER, P. Vegetation description analyses. London: Behaven Press, 1992. 363p.

KILKA, R. V. Alguns aspectos florísticos e estruturais de uma floresta de galeria no sul da Planície Costeira do Rio Grande do Sul. 2002. 74 p. Monografia (Bacharelado em Ciências Biológicas) - Universidade Federal de Pelotas, Pelotas, 2002.

KLEIN, R. M. Síntese ecológica da floresta estacional da bacia do Jacuí e importância do reflorestamento com essências nativas (RS). In: CONGRESSO FLORESTAL ESTADUAL, 5., Nova Prata, 1984. Anais... Nova Prata: Prefeitura Municipal de Nova Prata, 1984. v.2, p.265-278.

LONGHI, R. A. Livro das árvores: árvores e arvoretas do Sul. Porto Alegre: L \& PM, 1995. 176p.

LONGHI, S. J.; NASCIMENTO, A. R. T.; FLEIG, F. D.; DELLA-FLORA, J. B.; FREITAS, R. A. de; CHARÃO. L. W. Composição flrorística e estrutura da comunidade arbórea de um fragmento florestal no município de Santa Maria, Brasil. Ciência Florestal, v.9, n.1, p.115-133, 1999a.

LONGHI, S. J.; CANTARELLI, E. B.; MARAFIGA, J. A. S.; MATTOS, R. B. de. Aspectos florísticos da Floresta Estacional Decidual, às margens do Rio Ibicuí-Mirim no distrito de Boca do Monte, Santa Maria-RS. In: CICLO DE ATUALIZAÇÃO FLORESTAL DO CONE-SUL, 1., 1999, Santa Maria. Anais... Santa Maria: UFSM, 1999b. p.254261.

LONGHI, S. J.; ARAUJO, M. M.; KELlinG, M. B.; HOPPE, J. M.; MÜlleR, I.; BORSOI, G. B. Aspectos fitossociológicos de fragmento de Floresta Estacional Decidual, Santa Maria, RS. Ciência Florestal, v.10, n.2, p.59-74, 2000 .

LONGHI. S. J.; ARAUJO, M. M.; KRÜGEL, M.; RIBEIRO, S. B. F.; ESBER, L. M.; ALBERTI, L. F.; CARVALHO JUNIOR, L. A.; MATTOS, R. B. de; TEIXEIRA, I. F. Padrões de distribuição espacial de espécies florestais em fragmento de mata ciliar, São Pedro do Sul, RS, Brasil. In: CONGRESSO FLORESTAL ESTADUAL, 8., 2000, Nova Prata. Anais... Nova Prata: Prefeitura Municipal de Nova Prata, 2001. p. 549-555.

LORENZI, H. Árvores brasileiras: manual de identificação e cultivo de plantas arbóreas nativas do Brasil. Nova Odessa: Ed. Plantarum, 1998. 368p. v.1.

MACHADO, P. F. dos S.; LONGHI, S. J. Aspectos florísticos e fitossociológicos do Morro do Elefante, Santa Maria, RS. Revista de Ciências Rurais, v.20, n.3-4, p.261-280, 1990.

McCUNE, B.; MEFFORD, M. J. PC-ORD: multivariate analysis of ecological data. Version 3.0. Oregon: MjM Software Design, 1997. 47p.

MARCHIORI, J. N. C. Dendrologia das angiospermas: das bixáceas às rosáceas. Santa Maria: Ed. da UFSM, 2000. 240 p.

MORENO, J. A. Clima do Rio Grande do Sul. Porto Alegre: Secretaria da Agricultura, 1961. 42p.

MOSER, J. M. Solos. In: IBGE. Geografia do Brasil: Região Sul. Rio de Janeiro: 1990. p.151-187.

NASCIMENTO, A. R. T.; LONGHI, S. J.; ALVAREZ FILHO, A.; GOMES, F. S. Análise da diversidade florística e dos sistemas de dispersão de sementes em um fragmento florestas na região central do Rio Grande do Sul, Brasil. Napaea, n.12, p.49-67, 2000.

REITZ, P.; KLEIN, R. M.; REIS, A. Projeto Madeira do Rio Grande do Sul. Sellowia, n. 34-35, p. 1-525, 1983.

RODRIGUES, R. R.; NAVE, A. G. Heterogeneidade florística das matas ciliares In: RODRIGUES, R. R.; LEITÃOFILHO, H. de F. (Eds.) Matas ciliares: conservação e recuperação. São Paulo: Editora da Universidade de São Paulo, 2000. p.45-71.

RUNHAAR, J.; HAES, H. A. U. de. The use of site factors as classification characteristics for ecotopes. In: KLIJN, F. (Ed.) Ecossistem classification for environmental management. Netherlands: Klwer Academic Publishers, 1994. p.139-172.

SAMPAIO, A. B.; WALTER, B. M. T.; FELFILI, J. M. Diversidade e distribuição de espécies arbóreas em duas matas de galeria na micro-bacia do Riacho Fundo, Distrito Federal. Acta Botânica Brasileira, v.14, n.2, p.197-214, 2000.

SCHIAVINI, I. Estrutura das comunidades arbóreas de mata de galeria da Estação Ecológica do Panga (Uberlândia, MG). 1992. 139p. Tese (Doutorado em Ciência - Ecologia) - Universidade de Campinas,Campinas, 1992.

SILVA, L. C. da; LONGHI, S. J.; FLEIG, F. D. Composição florística e fitossociológica do componente arbóreo do 
"Ecomuseu da Quarta Colônia" - Silveira Martins. In: CONGRESSO ESTADUAL FLORESTAL, 7., 1992, Nova Prata. Anais... Nova Prata: Prefeitura Municipal de Nova Prata, 1992. p. 357-375. v.1.

SILVA, P. E. N. da. Florística, fitossociologia e nutrição mineral do cerrado sentido restrito no Complexo Xavantina- MT. 2002. 111p. Tese (Doutorado em Ecologia) - Universidade de São Paulo, São Paulo, 2002.

TABARELli, M.; MACHADO, P. F. dos S.; LONGHI, S. J. Aspectos florísticos de um trecho de mata ciliar do rio Ibicuí, nos Municípios de Alegrete e São Francisco de Assis, RS. In: CONGRESSO FLORESTAL ESTADUAL, 7. 1992, Nova Prata. Anais... Santa Maria: CEPEF/FATEC - UFSM, 1992. p.416-428.

VACCARO, S.; LONGHI, S. J.; BRENA, D. A. Aspectos da composição florística e categorias sucessionais do estrato arbóreo de três subseres de uma floresta Estacional Decidual, no município de Santa Tereza - RS. Ciência Florestal, v.9, n. 1, p. 1-18, 1999.

WALTER, B. M. T. Distribuição espacial de espécies perenes em uma mata de galeria inundável no Distrito Federal; forística e fitossociologia. 1995. 200p. Dissertação (Mestrado em Ecologia) - Universidade de Brasília, Brasilia, 1995. 